\title{
Generation of [La(peptide) $]^{3+}$ Complexes in the Gas Phase: Determination of the Number of Binding Sites Provided by Dipeptide, Tripeptide and Tetrapeptide Ligands.
}

By

Tujin Shi, K.W. Michael Siu and Alan C. Hopkinson ${ }^{*}$

Department of Chemistry and Centre for Research in Mass Spectrometry, York University, 4700

Keele Street, Toronto, Ontario, Canada M3J 1P3. 
Table 1S: Total energies (hartrees) and Cartesian coordinates for the optimized structures in Figure 4.

A$$
6
$$$$
8
$$$$
7
$$$$
1
$$$$
6
$$$$
6
$$$$
6
$$$$
8
$$$$
8
$$$$
1
$$

$E_{t}=-\mathbf{1 4 5 7 . 5 0 6 9 8 9 5}$

\begin{tabular}{rrr}
-0.749090 & 1.556549 & -1.332129 \\
-0.049381 & 0.527867 & -1.118050 \\
-0.422233 & 2.714425 & -0.772557 \\
-0.964136 & 3.539347 & -1.013175 \\
0.677263 & 2.927246 & 0.203699 \\
2.144125 & 2.861309 & -0.351282 \\
0.469152 & 2.116340 & 1.491671 \\
-0.109851 & 1.016266 & 1.598579 \\
1.005967 & 2.727726 & 2.507790 \\
0.909937 & 2.240235 & 3.350226 \\
0.557479 & 3.966508 & 0.522971 \\
3.442593 & -0.803118 & -0.578818 \\
2.929198 & -2.037433 & -0.402104 \\
3.668811 & -3.077692 & -0.812014 \\
1.704793 & -2.231740 & 0.154080 \\
4.637518 & -2.978266 & -1.087608 \\
\hline 3.278715 & -4.006664 & -0.888796 \\
-0.406996 & -1.147441 & 0.453813 \\
-2.044587 & 1.417929 & -2.154059 \\
-1.851336 & 0.732685 & -2.983215 \\
-2.393998 & 2.370691 & -2.555642 \\
-3.094454 & -0.377675 & -0.801580 \\
-2.166258 & -1.181422 & -1.101033 \\
-4.057295 & -0.821650 & 0.290036 \\
-4.730194 & -0.024892 & 0.615343 \\
-4.667732 & -1.642013 & -0.099206 \\
-3.176526 & -1.291716 & 1.410581 \\
-3.381467 & -0.743829 & 2.246883 \\
-3.439740 & -2.246453 & 1.658094 \\
-3.106079 & 0.869873 & -1.277930 \\
-3.900940 & 1.466333 & -1.066611 \\
4.314116 & -0.752324 & -1.093827 \\
3.031159 & 0.440610 & 0.076838 \\
\hline .732642 & 1.561587 & -0.937842 \\
\hline & & \\
\hline
\end{tabular}




$\begin{array}{rrrrr}1 & 0 & 2.108607 & 1.173669 & -1.743494 \\ 1 & 0 & 3.681148 & 1.855270 & -1.402484 \\ 1 & 0 & 3.825290 & 0.754868 & 0.765141 \\ 1 & 0 & 2.168024 & 0.201303 & 0.701055 \\ 1 & 0 & 1.632926 & -3.219209 & 0.410535 \\ 1 & 0 & 2.776999 & 3.232706 & 0.463010 \\ 1 & 0 & 2.185579 & 3.631574 & -1.127678\end{array}$

B

$E_{t}=-1457.5106481$

\begin{tabular}{|c|c|c|c|c|}
\hline 6 & $\odot$ & $-\odot .898842$ & 1. 223898 & -1.158070 \\
\hline 8 & $\odot$ & $-\odot .128708$ & ๑. 238051 & -0.964359 \\
\hline 7 & $\odot$ & -0.583942 & 2.442188 & $-\odot .734866$ \\
\hline 1 & $\odot$ & -1.237110 & 3.189430 & $-\odot .951251$ \\
\hline 6 & $\odot$ & 0.632119 & 2.874442 & -0.000647 \\
\hline 6 & $\odot$ & 1.979124 & $2.85400 \odot$ & -0.805439 \\
\hline 6 & $\odot$ & 0.734698 & 2.240576 & 1.390843 \\
\hline 8 & $\odot$ & $\odot .366377$ & 1.090799 & 1.706673 \\
\hline 8 & $\odot$ & 1.291146 & 3.055149 & 2.235663 \\
\hline 1 & $\odot$ & 1.392850 & 2.679396 & 3.133674 \\
\hline 1 & $\odot$ & $\odot .457659$ & 3.933953 & ๑. 205160 \\
\hline 7 & $\odot$ & 3.562128 & -0.701085 & -0.981075 \\
\hline 6 & $\odot$ & 3.112098 & -1.943611 & -0.718074 \\
\hline 7 & $\odot$ & 3.813792 & -2.971259 & -1.217877 \\
\hline 7 & 0 & 1.992728 & -2.157119 & $\odot .020121$ \\
\hline 1 & 0 & 4.718646 & -2.848163 & -1.653925 \\
\hline 1 & $\odot$ & 3.464439 & -3.918637 & -1.185377 \\
\hline 57 & 0 & -0.005133 & -1.145068 & ๑.868611 \\
\hline 6 & $\odot$ & -2.206676 & 1.012305 & -1.922353 \\
\hline 1 & $\odot$ & -1.956764 & 0.400373 & -2.797792 \\
\hline 1 & $\odot$ & $-2.6 \odot 4562$ & 1.962396 & -2.287922 \\
\hline 6 & 0 & -3.275571 & $-\odot .547197$ & $-\odot .209824$ \\
\hline 8 & 0 & -2.232317 & -1.004464 & 0.384007 \\
\hline 6 & 0 & -4.669765 & -1.041609 & ๑. 182976 \\
\hline 1 & $\Theta$ & -4.666591 & -2.131519 & ๑. . 058245 \\
\hline 1 & $\Theta$ & -4.792990 & $-\odot .85640 \odot$ & 1.256614 \\
\hline 7 & 0 & -5.672609 & -0.371721 & -0.633084 \\
\hline 1 & 0 & -6.198767 & -1.016577 & -1.216032 \\
\hline 1 & $\odot$ & -6.338559 & $\odot .159594$ & $-\odot . \odot 79737$ \\
\hline
\end{tabular}




$\begin{array}{ll}7 & 0 \\ 1 & 0 \\ 6 & 0 \\ 6 & 0 \\ 1 & 0 \\ 1 & 0 \\ 1 & 0 \\ 1 & 0 \\ 1 & 0 \\ 1 & 0 \\ 1 & 0 \\ 1 & 0\end{array}$

\section{C}

$$
E_{t}=-1457.5146036
$$

$\begin{array}{cc}6 & 0 \\ 8 & 0 \\ 7 & 0 \\ 1 & 0 \\ 6 & 0 \\ 6 & 0 \\ 6 & 0 \\ 8 & 0 \\ 8 & 0 \\ 1 & 0 \\ 1 & 0 \\ 7 & 0 \\ 6 & 0 \\ 7 & 0 \\ 7 & 0 \\ 1 & 0 \\ 1 & 0 \\ 57 & 0 \\ 6 & 0 \\ 1 & 0 \\ 1 & 0 \\ 6 & 0 \\ 8 & 0 \\ 6 & 0\end{array}$

\begin{tabular}{rrr}
-0.853970 & 1.334330 & -1.091449 \\
-0.163463 & 0.284660 & -0.922016 \\
-0.395493 & 2.523683 & -0.707559 \\
-0.993232 & 3.322980 & -0.891795 \\
0.900459 & 2.854219 & -0.070055 \\
2.185478 & 2.683624 & -0.953945 \\
1.033751 & 2.252523 & 1.331655 \\
0.555164 & 1.168096 & 1.715927 \\
1.745749 & 3.018242 & 2.106237 \\
1.862651 & 2.653947 & 3.006395 \\
0.843740 & 3.931085 & 0.111524 \\
3.423489 & -1.005726 & -1.085402 \\
2.880089 & -2.193796 & -0.750252 \\
3.469789 & -3.295376 & -1.243532 \\
1.787952 & -2.282009 & 0.043816 \\
4.363207 & -3.265263 & -1.716651 \\
\hline 3.041972 & -4.206235 & -1.160761 \\
-0.086456 & -1.044635 & 0.944102 \\
-2.217917 & 1.262089 & -1.776424 \\
-2.026875 & 0.822883 & -2.765755 \\
-2.595867 & 2.272660 & -1.961011 \\
-3.264094 & -0.361662 & -0.214490 \\
-2.266153 & -0.819703 & 0.518922 \\
-4.618295 & -0.984979 & 0.155034
\end{tabular}




$\begin{array}{lrrrr}1 & 0 & -4.586362 & -2.069207 & 0.029468 \\ 1 & 0 & -4.867095 & -0.768441 & 1.196128 \\ 7 & 0 & -5.681376 & -0.406510 & -0.745903 \\ 1 & 0 & -6.129987 & -1.109617 & -1.342217 \\ 1 & 0 & -6.422816 & 0.081755 & -0.233133 \\ 7 & 0 & -3.291272 & 0.506009 & -1.150580 \\ 1 & 0 & -5.155757 & 0.277539 & -1.346429 \\ 1 & 0 & 4.191653 & -1.031529 & -1.744892 \\ 6 & 0 & 3.148875 & 0.299233 & -0.488341 \\ 6 & 0 & 2.605245 & 1.310542 & -1.515046 \\ 1 & 0 & 1.797026 & 0.852851 & -2.086413 \\ 1 & 0 & 3.405767 & 1.519560 & -2.234253 \\ 1 & 0 & 4.073229 & 0.677835 & -0.035530 \\ 1 & 0 & 2.456312 & 0.133483 & 0.339608 \\ 1 & 0 & 1.674519 & -3.264446 & 0.304452 \\ 1 & 0 & 3.002465 & 3.124495 & -0.370645 \\ 1 & 0 & 2.033715 & 3.360246 & -1.800492\end{array}$

D

$$
E_{t}=-\mathbf{1 4 5 7 . 5 4 7 1 6 8 6}
$$

6

6

8

8

57

1

7

6

6

8

1

7

1

1

6

8

6

1

1

\section{$\odot$}

0

$\odot$

$\odot$

$\odot$

0

$\odot$

0

$\odot$

$\odot$

$\odot$

$\odot$

$\odot$

$\odot$

$\odot$

$\odot$

$\odot$

$\odot$

$\odot$
๑. 899411

$\odot .132300$

0.056576

$-0.642289$

$-2.359100$

1.106051

- 0.057909

$-1.323402$

$-2.411493$

$-1.667241$

$-2.034734$

$-2.947120$

0.242280

$-2.762477$

$-3.584683$

$-3.873922$

$-3.834448$

$-4.915599$

$-3.394893$
0.679257

$-0.473996$

$-1.615596$

$-0.195052$

$-1.363712$

$\odot .353846$

1.817848

1.610190

2.658176

0.473636

3.682080

2. 379376

2.729932

3. 054991

1. 254167

๑. 398998

0.822502

$\odot .793969$

1.499434
$-0.825764$

$-0.141036$

$-0.672993$

$\odot .852247$

$-0.268459$

$-1.849921$

$-0.874881$

$-1.230882$

$-0.940322$

$-1.662448$

$-\odot .951899$

$\odot .416160$

$-0.548373$

1.150413

$\odot .726856$

$-0.161262$

2.164694

2. 329607

2. 900746 


$\begin{array}{ll}7 & 0 \\ 1 & 0 \\ 1 & 0 \\ 1 & 0 \\ 6 & 0 \\ 1 & 0 \\ 1 & 0 \\ 6 & 0 \\ 1 & 0 \\ 1 & 0 \\ 6 & 0 \\ 1 & 0 \\ 1 & 0 \\ 7 & 0 \\ 6 & 0 \\ 7 & 0 \\ 7 & 0 \\ 1 & 0 \\ 1 & 0 \\ 1 & 0 \\ 1 & 0 \\ 1 & 0\end{array}$

$\begin{array}{rrr}-3.257940 & -0.558994 & 2.262771 \\ -2.437572 & -0.533885 & 2.869338 \\ -3.202134 & 2.559181 & -1.686946 \\ -3.929150 & -1.167524 & 2.733174 \\ 2.209943 & 1.100798 & -0.128105 \\ 2.522161 & 2.053991 & -0.573105 \\ 2.018125 & 1.282684 & 0.935918 \\ 3.344359 & 0.071701 & -0.313411 \\ 3.086034 & -0.875966 & 0.174413 \\ 3.470878 & -0.134802 & -1.383303 \\ 4.677400 & 0.589025 & 0.254041 \\ 4.585312 & 0.754181 & 1.335035 \\ 4.929799 & 1.545460 & -0.222426 \\ 5.750812 & -0.381681 & -0.000720 \\ 7.040762 & -0.220773 & 0.347774 \\ 7.435980 & 0.880442 & 0.993107 \\ 7.933020 & -1.171116 & 0.051708 \\ 5.518034 & -1.213943 & -0.524588 \\ 6.800434 & 1.629921 & 1.214477 \\ 8.399440 & 1.006885 & 1.270997 \\ 8.911464 & -1.066882 & 0.282924 \\ 7.671469 & -2.025265 & -0.418773\end{array}$

E

$$
E_{t}=-1457.551571
$$

$\begin{array}{rr}6 & 0 \\ 6 & 0 \\ 8 & 0 \\ 8 & 0 \\ 57 & 0 \\ 1 & 0 \\ 7 & 0 \\ 6 & 0 \\ 6 & 0 \\ 8 & 0 \\ 1 & 0 \\ 7 & 0 \\ 1 & 0 \\ 1 & 0\end{array}$

$\begin{array}{rrr}-1.011663 & 0.391187 & 0.810821 \\ -0.384928 & -0.745018 & -0.015218 \\ -0.365373 & -1.933905 & 0.418221 \\ 0.354326 & -0.430213 & -1.017590 \\ 2.006819 & -1.793370 & -0.081539 \\ -1.265516 & -0.020020 & 1.792757 \\ 0.071839 & 1.400418 & 0.986808 \\ 1.318208 & 1.069275 & 1.299683 \\ 2.396923 & 2.138185 & 1.375780 \\ 1.634340 & -0.138990 & 1.528025 \\ 1.970817 & 3.141899 & 1.298945 \\ 3.440954 & 2.030643 & 0.346669 \\ -0.149539 & 2.373380 & 0.802934 \\ 4.069308 & 2.861735 & 0.195760\end{array}$




\begin{tabular}{|c|c|c|c|c|}
\hline 6 & $\odot$ & 3.891947 & 1.080014 & $-\odot .438831$ \\
\hline 8 & $\odot$ & 3.422262 & -0.111486 & -0.561866 \\
\hline 6 & $\odot$ & 5.104259 & 1.491561 & -1.281043 \\
\hline 1 & $\odot$ & 4.829988 & 1.344994 & -2.332359 \\
\hline 1 & $\odot$ & 5.900249 & 0.768485 & -1.065995 \\
\hline 7 & $\odot$ & 5.477007 & 2.864633 & -0.974726 \\
\hline 1 & $\odot$ & 6.407800 & 2.938822 & -0.574543 \\
\hline 1 & $\odot$ & 2.863901 & 2.050923 & 2.364958 \\
\hline 1 & $\odot$ & 5.436715 & 3.473259 & -1.786693 \\
\hline 6 & $\odot$ & -2.249748 & 1.041591 & 0.157809 \\
\hline 1 & $\odot$ & -2.474466 & 1.959760 & $\odot .715005$ \\
\hline 1 & $\odot$ & -2.002842 & 1.331173 & -0.870389 \\
\hline 6 & $\odot$ & -3.488750 & 0.122543 & $\odot .185628$ \\
\hline 1 & $\odot$ & -3.281440 & $-\odot .803449$ & $-\odot .366611$ \\
\hline 1 & $\odot$ & -3.716896 & -0.160968 & 1.219226 \\
\hline 6 & $\odot$ & -4.720498 & 0.812724 & -0.428094 \\
\hline 1 & $\odot$ & -4.517622 & 1.086988 & -1.471934 \\
\hline 1 & $\odot$ & -4.947851 & 1.727986 & 0.128343 \\
\hline 7 & $\odot$ & -5.896779 & -0.064886 & -0.349336 \\
\hline 6 & $\odot$ & -7.180002 & 0.343239 & -0.341296 \\
\hline 7 & $\odot$ & -7.492598 & 1.618433 & $-\odot .594217$ \\
\hline 7 & $\odot$ & -8.146546 & -0.533664 & -0.052167 \\
\hline 1 & $\odot$ & -5.744732 & -1.060736 & $-\odot .445451$ \\
\hline 1 & $\odot$ & -6.870971 & 2.223950 & -1.108921 \\
\hline 1 & $\odot$ & -8.427801 & 1.964685 & -0.425496 \\
\hline 1 & $\odot$ & -9.118493 & -0.324267 & -0.236554 \\
\hline 1 & $\odot$ & -7.945334 & -1.420577 & ๑. 387751 \\
\hline
\end{tabular}

\section{F}

6

6

8

8

57

1

7

6

6

$E_{t}=-1457.5525107$

$\begin{array}{rrr}-0.988583 & 0.507317 & 0.907750 \\ -0.454852 & -0.664509 & 0.065566 \\ -0.464956 & -1.844383 & 0.518354 \\ 0.239652 & -0.391301 & -0.980303 \\ 1.910559 & -1.775330 & -0.090266 \\ -1.192720 & 0.117839 & 1.909965 \\ 0.131679 & 1.481595 & 0.998964 \\ 1.382828 & 1.115046 & 1.261202 \\ 2.500361 & 2.147126 & 1.240715\end{array}$




\begin{tabular}{|c|c|c|c|c|}
\hline 8 & $\odot$ & 1.655768 & -0.101692 & 1.509983 \\
\hline 1 & $\odot$ & 2.090342 & 3.152215 & 1.099350 \\
\hline 7 & $\odot$ & 3.572014 & 1.971259 & ๑. 269585 \\
\hline 1 & $\odot$ & -0.060690 & 2.455094 & $\odot .790677$ \\
\hline 6 & $\odot$ & 3.910075 & 0.980665 & $-\odot .461773$ \\
\hline 8 & $\odot$ & 3.389995 & $-\odot .222646$ & $-\odot .625989$ \\
\hline 6 & $\odot$ & 5.160519 & 1.198692 & -1.328534 \\
\hline 1 & $\odot$ & 4.908596 & 1.126333 & -2.388527 \\
\hline 1 & $\odot$ & 5.916791 & 0.444521 & -1.102459 \\
\hline 7 & $\odot$ & 5.711554 & 2.570554 & -1.033794 \\
\hline 1 & $\odot$ & 6.646248 & 2.547574 & $-\odot .614264$ \\
\hline 1 & $\odot$ & 2.951019 & 2.134869 & 2.242424 \\
\hline 1 & $\odot$ & 5.755383 & 3.175821 & -1.859217 \\
\hline 6 & $\odot$ & -2.248532 & 1.180591 & $\odot .327327$ \\
\hline 1 & $\odot$ & -2.417245 & 2.109947 & $\odot .885728$ \\
\hline 1 & $\odot$ & -2.062956 & 1.452564 & -0.718449 \\
\hline 6 & $\odot$ & -3.499948 & 0.287225 & 0.446917 \\
\hline 1 & $\odot$ & -3.371319 & -0.639595 & $-\odot .123768$ \\
\hline 1 & 0 & -3.634364 & $\odot .0 \odot 2776$ & 1.498630 \\
\hline 6 & $\odot$ & -4.767401 & 1.008135 & -0.043644 \\
\hline 1 & $\odot$ & -4.691622 & 1.214361 & -1.116400 \\
\hline 1 & $\odot$ & -4.879653 & 1.965138 & 0.483867 \\
\hline 7 & $\odot$ & -5.951831 & 0.165652 & 0.176831 \\
\hline 6 & $\odot$ & -7.131501 & $\odot .291606$ & -0.457578 \\
\hline 7 & $\odot$ & -7.358283 & 1.322616 & -1.278921 \\
\hline 7 & $\odot$ & -8.074425 & -0.640758 & -0.286233 \\
\hline 1 & $\odot$ & -5.939127 & $-\odot .449420$ & 0.980522 \\
\hline 1 & $\odot$ & -6.825520 & 2.176952 & -1.215061 \\
\hline 1 & $\odot$ & -8.176480 & 1.340455 & -1.872836 \\
\hline 1 & $\odot$ & -9.029235 & -0.482902 & -0.578266 \\
\hline 1 & $\odot$ & -7.866037 & -1.536476 & 0.131337 \\
\hline 1 & $\odot$ & $5.02 \odot 295$ & 2.958155 & $-\odot .33720 \odot$ \\
\hline
\end{tabular}

